\title{
Exploitation des numérisations pour l'analyse urbaine en contexte archéologique : réflexion méthodologique sur le site précolombien de Pachacamac
}

\section{Exploitation of digitization for urban analysis in an archaeological context: Methodological reflection on the pre-Colombian site of Pachacamac}

\author{
Alexandre Van Dongen ${ }^{1,3, *}$, Sébastien Lambot ${ }^{2}$, Peter Eeckhout ${ }^{3}$ et David Lo Buglio ${ }^{1,4}$ \\ ${ }^{1}$ Laboratoire AlICe, Faculté d'Architecture, ULB, Belgique \\ ${ }^{2}$ GPRLouvain, Earth and Life Institute, Environmental Sciences, UCLouvain, Belgique \\ ${ }^{3} \mathrm{CReA}$-Patrimoine, Faculté de Philosophie et Sciences sociales, ULB, Belgique \\ ${ }^{4}$ MAP-Gamsau, UMR 3495 CNRS / MCC, Marseille, France
}

\begin{abstract}
Résumé. Cet article a pour objectif d'apporter une réflexion méthodologique sur l'exploitation des numérisations d'un site archéologique de grande échelle. Basées sur un grand volume de données issues de relevés photogrammétriques et GPR aériens, ces numérisations sont bien souvent utilisées pour l'enregistrement de l'état du bâti au moment de l'acquisition ou la prospection archéologique. Notre approche propose d'étendre l'exploitation de ces types de données massives afin d'enrichir la connaissance des logiques de conception urbaine d'une « cité » dont il ne reste que certaines parties monumentales encore visibles aujourd'hui. En outre, les méthodes d'analyse urbaine s'appuient largement sur une compréhension occidentale de la ville qui est parfois très éloignée de celle que l'on rencontre en Amérique du sud avant l'arrivée des conquistadors. Au-delà des enjeux de connaissances spécifiques au terrain d'étude, l'ambition première de cet article est de comprendre comment l'apport et l'exploitation des technologies présentes et futures en matière de numérisation patrimoniale pourraient nous aider à accroître notre connaissance du passé. En somme se projeter dans le futur pour opérer un retour vers le passé.
\end{abstract}

Mots-clés. Numérisations, Données massives, Analyse urbaine, Archéologie, Architecture précolombienne, Relevé, Documentation du patrimoine bâti.

\footnotetext{
*Corresponding author: avdongen@ulb.ac.be
} 


\begin{abstract}
The present article aims to provide methodological reflection on the exploitation of digitization of a large-scale archaeological site. Based on a large volume of data collected by aerial photogrammetry and GPR (Ground Penetrating radar) surveys, these scans are often used for recording the building status at the time of acquisition or for archaeological prospecting (GPR). Our approach intends to extend the use of such big data in view to enhance our understanding in the logics of urban conception of a 'city' of which only a few monumental parts remain still visible today. Besides, methods of urban analysis are largely based on a western understanding of the city, which is at times far from that encountered in South America before the arrival of conquistadors. Beyond the challenging knowledge specific to the field of study, the primary goal of this article is to understand how the contribution and exploitation of the present and future technologies in heritage digitalization could help us to increase our knowledge of the past.
\end{abstract}

Keywords. Digitization, Big Data, Urban Analysis, Archaeology, PreColombian Architecture, Survey, Digital Heritage.

\title{
1. Introduction
}

La photogrammétrie, et plus particulièrement si on lui ajoute l'adjectif « aérienne », a connu depuis plusieurs années, grâce à la démocratisation des drones, une croissance exponentielle. Développés dans un premier temps pour le domaine militaire et la cartographie, les outils disponibles aujourd'hui offrent la possibilité pour toute personne détentrice d'un drone muni d'un capteur photographique de réaliser des acquisitions photogrammétriques à basse altitude. Ces nouveaux dispositifs d'acquisitions, issus de plusieurs champs disciplinaires (archéométrie, ingénierie, informatique, architecture, VGA, ...), fournissent une quantité de données que l'on peut qualifier de massives et dont la majorité des acteurs (cartographes, géomètres, archéologues, architectes ou encore simples pilotes de drone) ne se servent que partiellement. Ces données sont alors utilisées pour l'enregistrement de l'état actuel des édifices dans le but d'en faire le monitoring et/ou servir de socle métrique/morphologique pour l'élaboration d'hypothèses de restitution ou de valorisation du patrimoine. Dans ce contexte, la complexité des numérisations est souvent réduite à une sélection d'informations exploitables et rarement envisagée pour investiguer des propriétés du lieu non perceptibles visuellement (Collective, 2011).

Notre recherche ambitionne, de manière générale, de nouvelles pistes de réflexions pour l'exploitation d'un grand volume de données afin d'accroître notre connaissance des logiques de conception urbaine dans un contexte d'étude patrimoniale. Outre les méthodes d'analyses automatiques développées pour l'étude des propriétés morphologiques du bâti (Lo Buglio et al., 2015), les données issues du travail de numérisation pourraient également permettre d'analyser et de représenter le non-visible à l'échelle urbaine. Le terme est à prendre ici au sens large. Nous entendons dans ce contexte tout ce qui n'est pas visible à l'œil nu ou qui n'est pas immédiatement mesurable et qui fait sens dans l'analyse architecturale et urbanistique d'un lieu. Dans le cas spécifique qui nous concerne, l'étude du non-visible porte sur trois volets que nous développerons ultérieurement : 1) l'analyse de l'intervisibilité, 2) l'étude des flux et 3) les structures enfouies sous une certaine couche de sable (ou de terre). L'intervisibilité produit des informations quant à la disposition dans l'espace des différentes structures et leurs relations les unes par rapport aux autres, tandis que les flux permettent d'apporter une composante dynamique dans l'utilisation de 
l'espace. Le dernier point apparaît évident mais est indispensable pour pouvoir mener une analyse d'un site à l'échelle urbaine la plus juste et la plus complète.

\subsection{Le cas d'étude : le Sanctuaire de Pachacamac}

Le site archéologique monumental de Pachacamac est situé à environ $30 \mathrm{~km}$ au sud de Lima, sur la côte centrale du Pérou (Eeckhout, 1999). Ce sanctuaire apparaît comme l'un des sites les plus importants des Andes Centrales avec une superficie de près de 600 hectares et une occupation permanente qui s'étale sur plus de mille ans (Ve XVIe s. CE). Quatre cultures (Lima, Wari, Ychsma, Inca) s'y sont succédé et ont chacune apporté leur lot de constructions.

Les premières représentations du sanctuaire datent de 1793, mais la connaissance du lieu se construit principalement par une succession de campagnes de fouilles entreprises depuis 1896 par Max Uhle (Uhle, 1903). Plusieurs plans de la partie monumentale ont été dressés mais aucun ne détaille le site au-delà de cette zone. Pourtant, Pachacamac comprend quatre enceintes concentriques. La première est la plus sacrée, avec des édifices voués aux cultes. La deuxième enceinte est réservée en partie à l'élite et aux pèlerins avec de nombreuses pyramides à rampe. La troisième semble servir d'espace tampon et accueille des structures publiques, un cimetière et d'autres constructions basses qui sont aujourd'hui totalement ensevelies. C'est en périphérie de cette zone que se situe la porte monumentale du site de Pachacamac. Enfin, à environ $1 \mathrm{~km}$ de la 3ème enceinte se situe une 4ème qui forme les faubourgs.
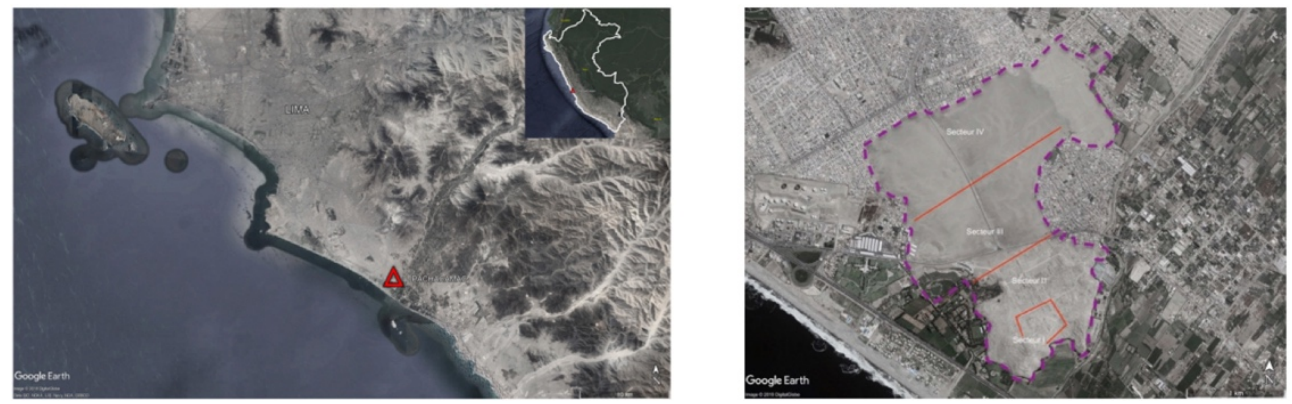

Figure 1. Localisation du site de Pachacamac et ses différents secteurs.

Depuis le début des fouilles en 1896, les archéologues se concentrent principalement sur les deux enceintes les plus sacrées du site que sont les secteurs I et II (Eeckhout, 1999 ; Uhle, 1903 ; Shimada et al., 2004). C'est en effet dans ces zones qu'apparaît le bâti encore visible aujourd'hui et c'est là que se seraient pratiqué tous les rituels et autres sacrifices. Mais pour étudier Pachacamac en tant que «ville », il est nécessaire de mener des analyses sur le tissu urbain au-delà de ces deux enceintes. Malgré l'inscription de ce complexe archéologique à la liste de l'héritage culturel de l'UNESCO, la pression immobilière à Lima met en danger la préservation du site. Il y a donc une certaine urgence à extraire du site un maximum de données.

Le cas de Pachacamac apparaît intéressant à plusieurs égards. En effet, aucun document écrit ou iconographique n'a été réalisé par les bâtisseurs, nous sommes donc face à un site qui est lui-même sa seule source de connaissances, hormis les quelques descriptions parfois enjolivées des textes ethno-historiques. Grâce aux fouilles, il est aujourd'hui possible d'émettre des hypothèses sur l'évolution chronologique des bâtiments situés dans la partie monumentale du site. Mais de nombreuses questions subsistent sur les rapports qui 
existaient entre les différents édifices ainsi que sur l'organisation des flux de pèlerins à travers des parcours conçus à l'échelle du site et du territoire (Eeckhout, 2013).

Cet article porte donc sur une réflexion méthodologique visant à exploiter les données massives acquises lors de campagnes toujours en cours à Pachacamac. En faisant appel aux méthodes de numérisation les plus récentes que sont la photogrammétrie aérienne et le GPR monté sur drone, nous possédons des informations susceptibles d'accroître notre connaissance de ce sanctuaire précolombien.

\section{Réflexion méthodologique}

Dans notre définition du non-visible nous mettons en avant trois pistes de réflexion pour l'exploitation de données massives issues d'acquisitions par drone : l'analyse de l'intervisibilité, l'étude des flux et les structures enfouies. Les deux premières pistes sont très proches et intègrent des théories issues de la syntaxe spatiale (Hillier et Hanson, 1984 ; Turner et al., 2001). Nous prenons ici le parti de les scinder pour clarifier notre méthodologie.

Le recours à l'analyse par graphes de visibilité, ou à la syntaxe spatiale de manière générale, n'est pas neuf en architecture, en urbanisme ou en archéologie. Ce type d'analyse est utilisé pour comprendre et quantifier les relations qui existent entre différents espaces d'une même entité (bâtiment, structure urbaine, ...). De nombreux cas d'études ont été réalisés, notamment sur des sites archéologiques situés en Amérique du Sud. Wernke (2012) a étudié grâce à l'analyse des réseaux spatiaux l'évolution des cheminements à travers un ancien comptoir impérial inca devenu colonie espagnole. Nous pouvons également citer les travaux de Edwards (2013) qui utilise la syntaxe spatiale pour analyser les accès et décrire les relations entre le site de Pataraya (Nazca) et une petite structure Wari.

Bien souvent l'analyse par graphe de visibilité se base sur des plans, des dessins, des photographies d'époque ou des bâtiments en relativement bon état de conservation. Dans le contexte qui nous occupe, il n'existe aucun plan dressé par les bâtisseurs, la partie monumentale est en ruine et la plupart des murs n'ont plus leur hauteur originelle. De plus, le nombre d'édifices présents aujourd'hui dans la partie monumentale du sanctuaire correspond à l'arrivée des espagnols. Or, comme énoncé plus haut, Pachacamac a connu pas moins de quatre cultures et donc autant de changements urbanistiques. L'originalité de notre approche tient dans la nature des données dont nous disposons. Grâce aux acquisitions photogrammétriques par drone, nous disposons d'un modèle numérique du site de Pachacamac. Si nous désirons dresser un graphe d'intervisibilité ou une carte des flux qui correspond à un moment de l'évolution du site, nous pouvons opérer une sélection des données et ne conserver que les bâtiments contemporains de cet instant. Cela suppose d'enrichir la numérisation de l'état actuel par des hypothèses de restitutions basées sur les découvertes archéologiques et les quelques textes ethno-historiques.

\subsection{L'intervisibilité}

L'intervisibilité est le rapport visuel entre deux points. Elle dépend de l'échelle des points mis en relation et de la distance qui les sépare (Ogburn, 2006). En effet, une personne qui se tient dans les collines à plusieurs kilomètres de Pachacamac peut sans difficulté voir les constructions, mais à l'inverse il est impossible que cette même personne soit vue depuis le haut d'un temple situé au centre du site. Plusieurs niveaux d'intervisibilité doivent être définis suivant leur pertinence pour l'analyse urbanistique de 
Pachacamac. Il s'agit dans ce contexte d'établir de nouvelles clés de lecture pour appréhender de manière quantitative les rapports sociaux, hiérarchiques, les rapports au sacré ou encore de vérifier le statut, la finalité d'un bâtiment. En introduisant la notion de temporalité, en analysant l'évolution de ces relations au fil des différentes occupations, nous pouvons observer un éventuel changement de statut du sanctuaire vers celui de ville.

Deux niveaux d'intervisibilité sont ici définis : 1) le site dans son contexte et 2) les liens visuels au sein du site.

1. Un ensemble urbain s'insère dans un contexte et possède des liens (ressources, protections par rapport au menaces, facilités d'accès, culturels ou croyances) plus ou moins importants avec celui-ci. En étudiant les rapports d'intervisibilité qui apparaissent entre une ville (ou autre forme urbaine) et son environnement géographique, nous pouvons approfondir la connaissance sur son édification. Pachacamac entretient des relations importantes avec son environnement immédiat, aussi bien du point de vue de la proximité des ressources que des interactions visuelles avec le contexte paysager. Une meilleure compréhension de ces rapports permettrait d'approfondir la connaissance au niveau de la « scénographie » du lieu.

2. En nous intéressant au rapport d'intervisibilité qui existe entre les différents bâtiments nous pouvons - ou non - mettre en évidence une certaine logique de planification urbaine du site. Pour ce faire, il est important de considérer uniquement le bâti correspondant à un moment précis. En comparant les résultats de l'analyse des relations visuelles propres à chaque période (celle de l'Empire inca ou celle de la civilisation Ychsma), nous pourrons alors mieux comprendre le processus mis en place par les incas pour la planification de Pachacamac.
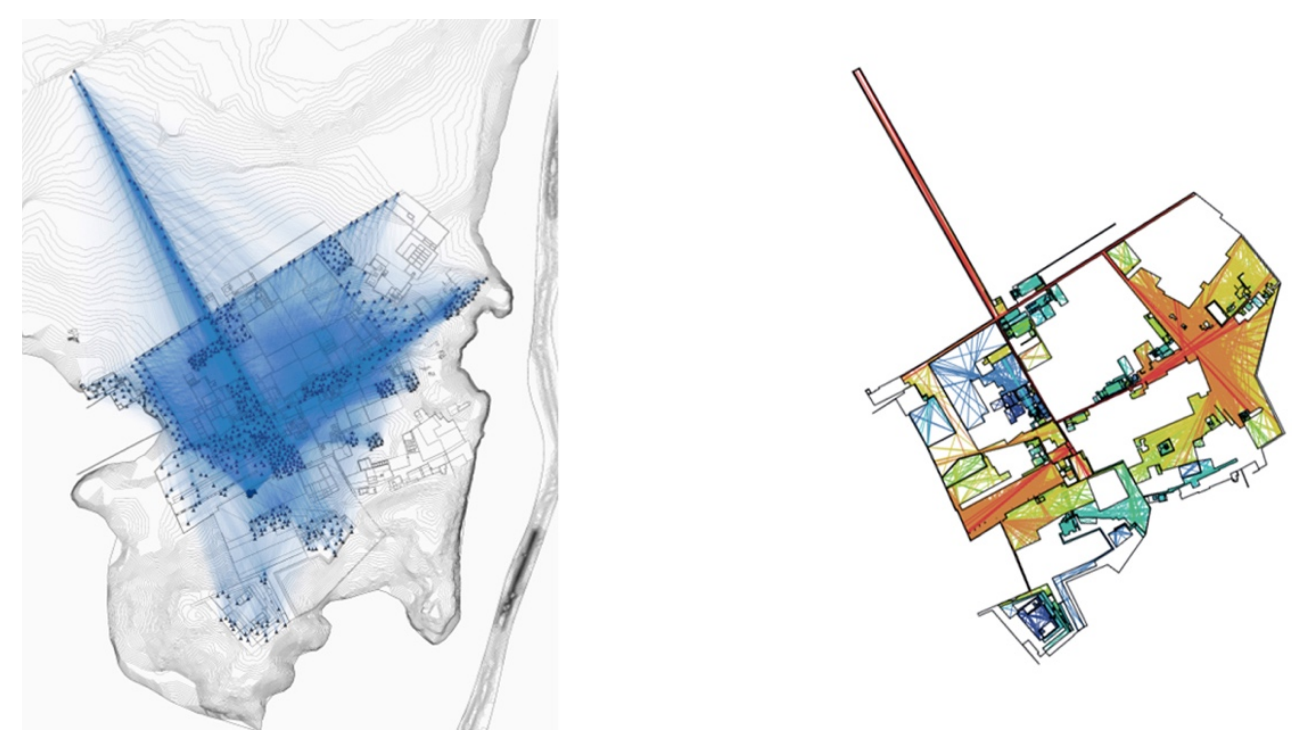

Figure 2. Exemple de carte d'intervisibilité (gauche) et de carte axiale (droite). Image : M. Jonckheere

En ce qui concerne les outils disponibles pour l'analyse de l'intervisibilité, notre choix se tourne vers deux logiciels open-source relativement complet et qui fournissent des cartes et graphes de visibilité interprétables : QGIS et DethmapX.

Le premier outil utilisé est QGIS. Il s'agit d'un logiciel de système d'information géographique et qui possède le plugin Viewshed Analysis développé par Zoran Čučković. 
Ce module d'extension offre la possibilité, sur base d'un raster MNT (Modèle Numérique de Terrain) de mener des analyses quantitatives du champ visuel depuis un point, du champ visuel d'un zone ou encore, l'intervisibilité entre différents points. La hauteur du point d'observation est ici prise en compte dans les calculs ce qui permet de limiter les erreurs.

Le second est développé par le Space Syntax Laboratory de The Bartlett, University College London et autorise la création de différents types de cartes à différentes échelles. Cependant, le logiciel ne semble travailler qu'en deux dimensions, ce qui peut créer dans notre cas des zones qui semblent être des espaces fermés alors qu'ils sont en réalité accessibles aussi bien visuellement que physiquement.

\subsection{Les flux}

L'analyse des flux est à distinguer de l'intervisibilité. Nous considérons en effet qu'un flux possède une composante dynamique, de mouvement et permet des liens physiques tandis que l'intervisibilité se base uniquement sur des liens visuels.

Dans les sociétés précolombiennes les déplacements s'opéraient uniquement à pied (elles ne connaissaient pas la roue et ne voyageaient pas à dos d'animaux). Le statut des «routes» au sein des villes n'était pas celui que nous connaissons dans l'Europe antique. Depuis sa création, Pachacamac était un centre religieux vers lequel convergeaient donc à pied de nombreux pèlerins. L'importance du site rayonnait sur de grandes distances et les gens y venaient parfois de très loin. Comprendre les déplacements, les parcours possibles dans cet imbroglio d'édifices et de places permet d'approfondir la connaissance sur le fonctionnement urbanistique du site. Pachacamac est structurée sur deux axes principaux, la rue nord-sud et la rue est-ouest. On y retrouve également le sentier touristique mais ce dernier n'existait évidemment pas à l'époque préhispanique. Pour le reste, il n'est pas évident de distinguer les parcours qu'empruntaient les pèlerins, les seigneurs ou autres prêtres pour se déplacer entre les bâtiments ni parfois au sein même des édifices.

L'étude des flux se fait en deux temps ; il faut d'abord distinguer les parcours possibles et ensuite les caractériser. Bien que certains sont facilement observables sur une orthophotographie, d'autres le sont nettement moins compte tenu de l'aspect uniforme du site (adobe \& désert). L'analyse des courbures de surfaces (c'est-à-dire l'expression mathématiques des courbures parcourant une surface pour rendre compte de ses variations) nous permet d'identifier les surfaces planes ainsi que ses limites non-perceptibles. Ces informations sont susceptibles de nous révéler la présence d'une voirie bordée par un bâti aujourd'hui effacé en partie. Il devient alors possible de simuler et de quantifier les flux sur le site en termes de parcours, de fluidité circulatoire et de leur importance dans le réseau.

Deux échelles de parcours sont à mettre en évidence : 1) les accès et sorties du site et 2) les parcours intérieurs.

1. Pachacamac est, comme nous l'avons vu précédemment, situé sur la côte centrale du Pérou. Le sanctuaire était relié au fameux Qhapaq Nan, réseau de routes inca de plus de $30000 \mathrm{~km}$ qui permettait des échanges en tous genres entre les différentes parties de l'Empire. En mettant en évidence le statut des différents flux (pèlerins, logistique, ...), nous envisageons de retracer le ou les parcours d'entrée et de sortie de Pachacamac afin de mieux apprécier son rayonnement. 
2. Pachacamac semble avoir accueilli un grand nombre de personnes au statut social varié (pèlerins, prêtres, seigneurs, etc.) qui devaient emprunter des parcours distincts suivant leur appartenance sociale. Nous pensons donc que l'analyse des flux à l'échelle du site apporterait un éclaircissement quant à la destination fonctionnelle des différents édifices.

Le principal outil utilisé pour l'étude des flux est inclus dans le logiciel DethmapX cité précédemment. Il s'agit de l'option wayfinding qui permet une simulation des déplacements. Encore une fois, le fait que le software ne prend pas en compte la dimension verticale, les résultats peuvent être erronés. Le développement de ces premiers outils seraient ici nécessaires pour limiter les erreurs et pouvoir se baser sur un modèle en trois dimensions.

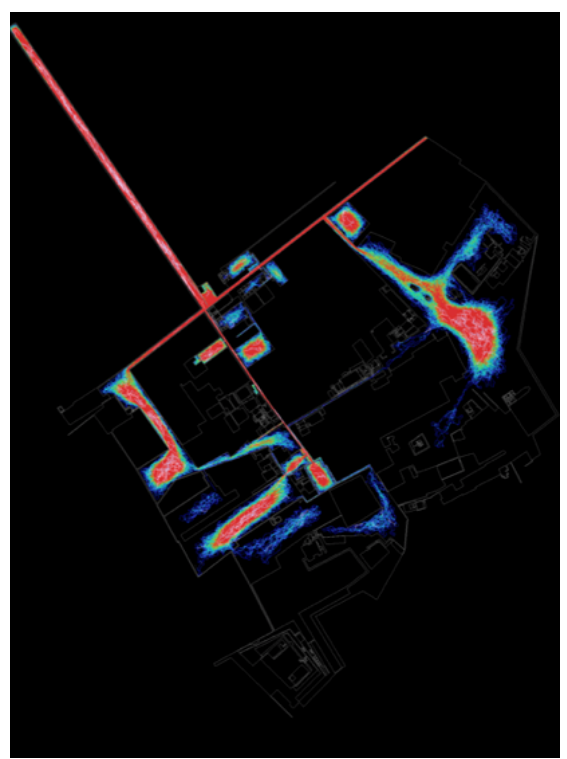

Figure 3. Carte des flux montrant en rouge les parcours les plus utilisés. Image : M. Jonckheere

\subsection{Les structures enfouies}

Bien souvent en archéologie les informations se trouvent encore enfouies. Les archéologues se concentrent alors sur des portions du territoire surtout pour les sites de grande superficie. Mais analyser un ensemble urbain par l'étude de la syntaxe spatiale suggère que nous possédions des données sur la totalité de sa surface.

Plusieurs techniques existent pour sonder le sous-sol et distinguer les structures enfouies (étude des micro-reliefs, géophysique, imagerie thermique, etc.). Au moment d'écrire cet article, nous en avons testé deux : 1) Photogrammétrie aérienne à basse altitude et 2) GPRaérien (toujours en phase de test).

1. L'acquisition par photogrammétrie aérienne à basse altitude permet d'obtenir des numérisations précises exploitables, notamment grâce à des fonctions algorithmiques développées dans Matlab afin d'étudier les micro-reliefs du site. En générant des cartes de courbure de surface (de la même manière que pour localiser les parcours au sein du site) nous pouvons distinguer des murs qui n'apparaissent que peu ou pas du tout à l'œil nu. Cette technique bien que rapide, atteint vite ses limites et nous oblige bien souvent à vérifier sur le terrain si nous avons bien affaire à une structure bâtie.
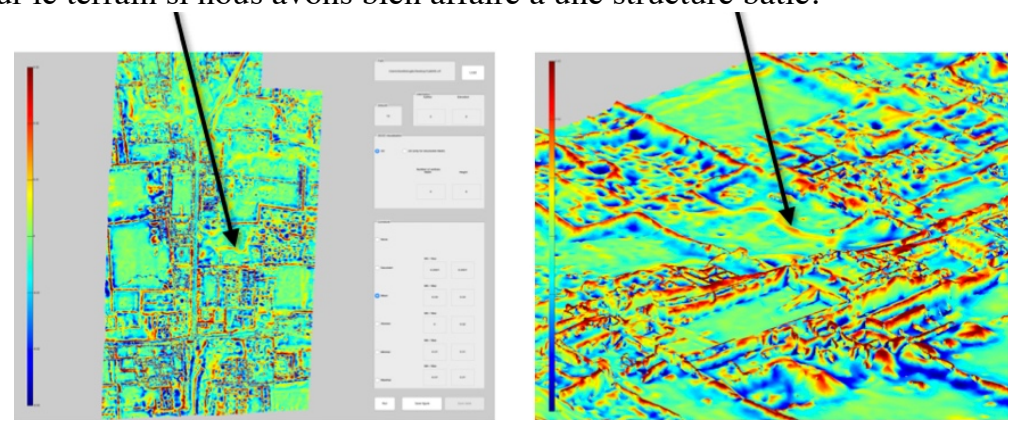

Figure 4. Détection de murs sur des cartes de courbure de surface. 
2. La technique du GPR (Ground Penetrating Radar) semble apporter des informations sur les structures en sous-sol. Bien que très répandue en prospection, cette technique d'acquisition de données a déjà été utilisée pour mener des analyses de syntaxe spatiale. Nous pouvons citer le cas du site archéologique de Doura-Europos en Syrie (Benech, 2007) ou celui de Tiwanaku en Bolivie (Spence-Morrow, 2009). À Pachacamac les 2/3 du site sont actuellement enfouis sous le sable. Depuis le début des fouilles en 1896, les archéologues se sont principalement intéressés à la partie monumentale du site. Nous ne possédons pas beaucoup de données concernant la structure urbaine du site au-delà de la 2ème enceinte et excaver 400 hectares est tout bonnement impensable. La technique du GPR a déjà été mise en place à Pachacamac (Shimada et al., 2004), mais elle semble plus être utilisée dans un but de prospection que véritablement de base pour l'analyse urbanistique du site. Le développement des drones ces dernières années permet aujourd'hui d'imaginer d'y monter des radar type GPR afin de rendre cette technique d'acquisition envisageable pour de grandes surfaces.

Indépendamment des tests que demande ce type de nouvel outil de relevé, nous avons élaboré des tests à échelle réduite en laboratoire. L'Université libre de Bruxelles a réalisé trois campagnes de fouilles sur l'édifice B15 du site de Pachacamac. Il s'agit dès lors d'un bâtiment dont nous connaissons la structure et que nous avons pu numériser (les bâtiments sont systématiquement remblayés après chaque campagne de fouilles dans un souci de conservation). Afin de pouvoir comparer et appréhender les résultats, aussi bien en laboratoire que sur le terrain, nous avons choisi cet édifice pour exécuter les premiers essais.

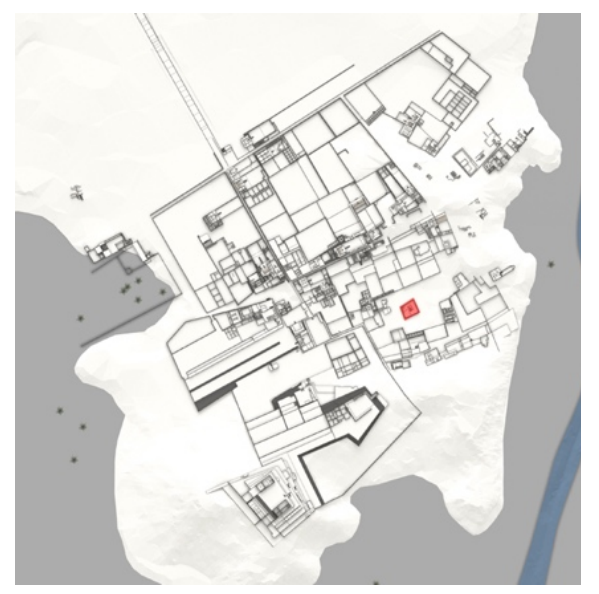

Figure 5. Localisation de l'édifice B15 (rouge).

Ainsi, à partir de la numérisation précise du B15, nous avons réalisé un modèle réduit (échelle 1/50) en matériau similaire à celui utilisé pour sa construction (argile sableuse). Ce modèle a ensuite été enfoui dans un bac à sable disponible au laboratoire GPRLouvain de l'UCLouvain.
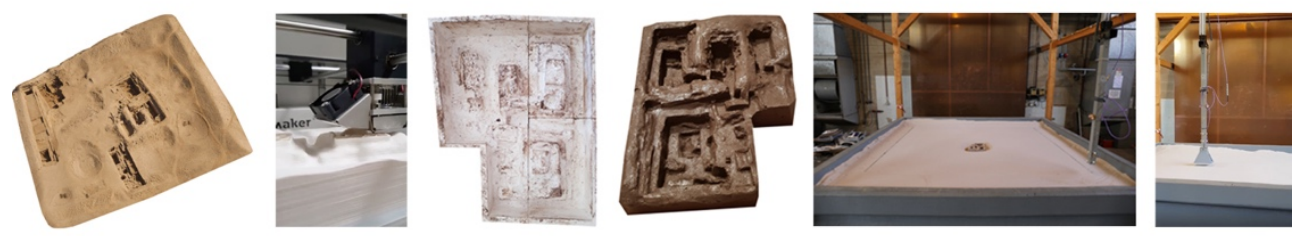

Figure 6. De gauche à droite : modèle 3D, impression d'un moule, moulage de la maquette en argile sablonneuse, mise en place de la maquette dans le bac à sable, acquisition par le GPR.

Nous avons mis en place un radar à pénétration de sol à ondes continues et fréquences étagées à l'aide d'un analyseur de réseau vectoriel connecté à une antenne cornet à double crête faisant office d'émetteur et de récepteur. Les mesures radar ont été effectuées automatiquement sur le bac à sable à l'aide d'une table de positionnement motorisée. L'espacement entre les mesures était de $1 \mathrm{~cm}$ dans les deux directions $\mathrm{x}$ et y. L'antenne était maintenue à $5 \mathrm{~cm}$ au-dessus de la surface de sable. Afin d'améliorer les images radar, les 
effets d'antenne en champ lointain ont été filtrés à l'aide du modèle radar de Lambot et al. (2004;2014). La figure 7 montre un exemple de profil radar traversant la construction enterrée. L'intégration des profils radar fournit un modèle tridimensionnel des réflexions, à partir duquel des tranches horizontales peuvent être extraites.

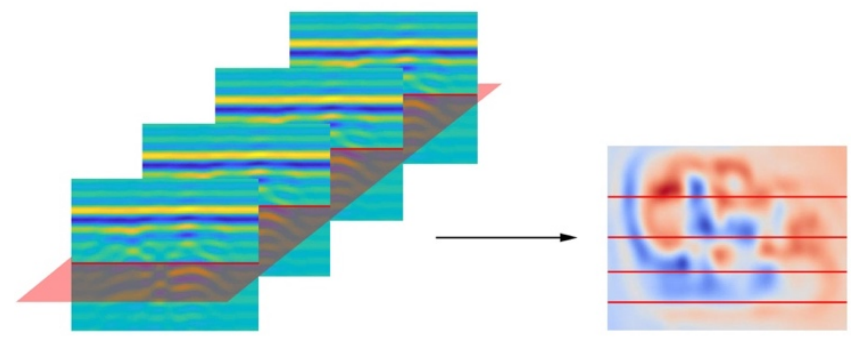

Figure 7. Principe envisagé pour le traitement et la transformation des données GPR. À gauche plusieurs profils radars verticaux qui permettent d'approcher un modèle 3D (transfert en coupe horizontale à droite) des réflexions en fonction de la nature des matériaux enfouis.

Lors de la dernière campagne de relevé en mars 2019, nous avons effectué un test sur la même zone de l'édifice B15. Pour des questions logistiques nous n'avons pas pu emporter un drone suffisamment grand pour embarquer un petit analyseur vectoriel et son antenne (prototype développé par le laboratoire GPRLouvain) mais nous avons néanmoins réalisé des acquisitions à environ $50 \mathrm{~cm}$ de hauteur par rapport à la surface grâce à une perche sur laquelle était fixé le GPR. Les données sont toujours en cours de traitement mais les premiers résultats, des coupes de l'édifice au droit de chaque passage du dispositif, montrent le bon fonctionnement du prototype.

\section{Conclusions}

Bien souvent, l'analyse urbaine par la syntaxe spatiale se base sur un grand nombre de sources (sources documentaires et/ou sources issues de campagnes de relevés). Il est donc logique de trouver ce type d'analyse pour des ensembles urbains pour lesquels on dispose d'une base de connaissances solides, comme c'est le cas des villes occidentales contemporaines ou antiques. Dans le cas qui nous occupe, l'absence de documentation d'époque et le faible pourcentage d'édifices encore visibles rendent plus difficile la compréhension des logiques de conception urbanistique mises en place par les sociétés précolombiennes.

La réflexion méthodologique énoncée dans cet article ouvre de nouvelles possibilités pour l'utilisation des données massives issues du relevé par drone. En effet, les techniques actuelles d'acquisitions permettent d'obtenir des informations jusqu'alors très peu utilisées dans l'analyse des structures urbaines, notamment les données GPR. À Pachacamac, une question supplémentaire que nous pouvons poser est de savoir si le site était considéré comme une véritable ville, pas seulement au regard de son développement sociale ou économique mais en termes d'organisation du territoire. Pour ce faire, il faut pouvoir comprendre l'entièreté du site, au-delà de la 2ème enceinte. C'est pourquoi l'une des perspectives de notre approche méthodologique porte sur la visualisation et l'analyse de structures enfouies.

L'arrivée du numérique au sein des pratiques de l'architecture et plus particulièrement au niveau de la documentation du fait bâti a engendré d'importants bouleversements. En outre, cette recherche vise à discuter et mettre en tension la dimension cognitive propre au relevé d'architecture avec les enjeux technologiques auxquels il fait face. Sur cette base, un constat s'impose : les empreintes numériques issues de l'application des méthodes 
d'acquisition les plus récentes expriment une forte exhaustivité (métrique et visuelle) avec les artefacts relevés mais produisent également d'importants volumes de données. Cette «surcharge d'informations» ne permet pas ou peu de renforcer la représentation architecturale dans son rôle de véhicule de connaissances. Face à ce déficit d'intelligibilité, notre ambition est de fournir une réponse épistémologique permettant de considérer ce besoin d'intelligibilité sans tourner le dos à la richesse des numérisations produites.

Nous tenons à remercier Denise Pozzi-Escot et le personnel du Musée de Site de Pachacamac pour leur aide ainsi que pour les facilités d'accès sur le terrain dans le cadre de notre projet de recherche.

\section{Bibliographie}

Benech, C. (2007). New approach to the study of city planning and domestic dwellings in the ancient Near East. Archaeological Prospection, 14(2), 87-103.

Collective (2011) Le relevé en architecture ou l'éternelle quête du vrai. Lieux Dits. Lyon.

Edwards, M. J. (2013). The configuration of built space at Pataraya and Wari provincial administration in Nasca. Journal of Anthropological Archaeology, 32(4), 565-576.

Eeckhout, P. (1999). Pachacamac durant l'Intermédiaire récent : étude d'un site monumental préhispanique de la Côte centrale du Pérou. British Archaeological Reports International Series, 747. K. Elissa, "Title of paper if known," unpublished.

Eeckhout, P. (2013). Dans les pas des Incas. Architecture précolombienne et syntaxe de l'espace. Clara (Bruxelles), (1), 61-79.

Hillier, B., \& Hanson, J. (1984). The social logic of space, 1984. Cambridge: Press syndicate of the University of Cambridge.

Lo Buglio, D., Lardinois, V., \& De Luca, L. (2015, February). What do thirty-one columns say about a "theoretical" thirty-second? ACM Journal on Computing and Cultural Heritage, 8(1), 6. doi:10.1145/2700425

Lambot, S., Slob, E.C., van den Bosch, I., Stockbroeckx, B. and Vanclooster, M. (2004) Modeling of ground-penetrating radar for accurate characterization of subsurface electric properties. IEEE Transactions on Geoscience and Remote Sensing 42(11), 2555-2568.

Lambot, S. and André, F. (2014) Full-Wave Modeling of Near-Field Radar Data for Planar Layered Media Reconstruction. IEEE Transactions on Geoscience and Remote Sensing 52(5), 2295-2303.

Ogburn, D. E. (2006). Assessing the level of visibility of cultural objects in past landscapes. Journal of Archaeological Science, 33(3), 405-413.

Shimada, I., Segura Llanos, R., Rostworowski De Diez Canseco, M., \& Watanabe, H. (2004). Una nueva evaluación de la Plaza de los Peregrinos de Pachacamac : raportes de la primera campaña 2003 del Proyecto Arqueológico Pachacamac. Bulletin de l'Institut français d'études andines, (33 (3)), 507-538.

Spence-Morrow, G. (2009). Analyzing the invisible syntactic interpretation of archaeological remains through geophysical prospection.

Turner, A., Doxa, M., O'sullivan, D., \& Penn, A. (2001). From isovists to visibility graphs: a methodology for the analysis of architectural space. Environment and Planning B: Planning and design, 28(1), 103- 121.

Uhle, M. (1903). Pachacamac: Report of the William Pepper, MD, LL. D., Peruvian Expeditions of 1896; Plan of the City and Twenty-one Plates in Phototype. Department of Archaeology of the University of Pennsylvania.

Wernke, S. A. (2012). Spatial network analysis of a terminal prehispanic and early colonial settlement in highland Peru. Journal of Archaeological Science, 39(4), 1111-1122. 